\title{
Comparative study of neural differentiation of bone marrow mesenchymal stem cells by different induction methods
}

\author{
M.W. Mu, Z.Y. Zhao and C.G. Li \\ Department of Orthopedics, \\ Dongzhimen Hospital Beijing University of Chinese Medicine, Beijing, China \\ Corresponding author: C.G. Li \\ E-mail: lichungen_bj@126.com \\ Genet. Mol. Res. 14 (4): 14169-14176 (2015) \\ Received May 7, 2015 \\ Accepted August 21, 2015 \\ Published October 29, 2015 \\ DOI http://dx.doi.org/10.4238/2015.October.29.39
}

\begin{abstract}
Neurogenic differentiation of bone marrow (BM) mesenchymal stem cells (MSCs) offers a new hope for patients with many neurological disorders. Several chemical inducers are employed to induce BMMSCs differentiation into nerve cells. In the present study, we compared different inducers [2-mercaptoethanol (BME), tretinoin (ATRA), dimethyl sulfoxide/ butylated hydroxyanisole (DMSO/BHA), and indomethacin/3-isobutyl-1methylxanthine (indomethacin/IBMX)] on the neurogenic differentiation of BMMSCs and aimed to identify a more efficient and safer method. The MSCs were first identified by their ability to adhere to plastic and by the expression of positive (CD44, CD90, and CD105) and negative (CD34) markers assessed by flow cytometry. The efficiency of the neurogenic differentiation was determined by assessing the mRNA and protein expression of nestin, microtubule-associated protein-2 (MAP2), neuron specific enolase (NSE), and glial fibrillary acidic protein (GFAP) by reverse transcription-polymerase chain reaction and western-blot, respectively. The effect of these inducers on cell viability was also evaluated by 3-(4,5-dimethylthiazol-2-yl)-2,5-diphenyltetrazolium bromide assay. This comprehensive study shows that indomethacin/IBMX is better than BME,
\end{abstract}


DMSO/BHA, and ATRA both in terms of efficiency and safety, while BME suppressed the growth and proliferation of MSCs.

Key words: Marrow mesenchymal stem cells; Neural differentiation; Induction

\section{INTRODUCTION}

Mesenchymal stem cells (MSCs) are an attractive cell source for cell therapy and regeneration. MSCs can be isolated and expanded from different adult tissues, including bone marrow (BM). Bone marrow mesenchymal stem cells are easily obtained from various sources and present a strong proliferationcapacity (Tatullo et al., 2015). In recent years, mesenchymal stem cells have been induced to differentiate into specific neurons such as cholinergic neurons, dopaminergic neurons, amino acid neurons, and peptidergic neurons (Woodbury et al., 2000; Fukuda, 2001; Notaraand Ahmed, 2012; Wu et al., 2013; Kristjanssonand Honsawek, 2014; Swart and Wulffraat, 2014; Zhang et al., 2014). Recent studies showed that bone marrow mesenchymal stem cells can survive in the damaged brain tissue and spinal cord, proliferate, migrate, and differentiate into neuron-like cells, thereby effectively improving the neurological function and survival status of patients following spinal cord injuries, stroke, and other nervous system diseases (Widgerow et al., 2013; Chen et al., 2014; Mizukami and Yagihashi, 2014).

Induction of adult rat BM MSCs by chemical compounds leads to neural differentiation and this protocol has been broadly used worldwide mainly for the following two intriguing aspects. First, MSCs acquire morphological features and express specific markers of mature neurons in a short time (e.g., Nestin, MAP2, NSE, and GFAP). Secondly, the percentage of differentiated neuronal cells is higher than when using other induction methods. However, the efficiency and safety of the chemical induction method are still controversial and experiments designed to determine the ideal conditions to obtain neural cells from MSC in vitro are still conducted worldwide. In the present study, several chemicals and neurotrophic factors were employed to induce mesenchymal stem cells into nerve cells such as 2-mercaptoethanol (BME), tretinoin (ATRA), dimethyl sulfoxide/butylatedhydroxyanisole (DMSO/BHA), and indomethacin/3-isobutyl-1-methylxanthine (Indomethacin/IBMX). The efficiency and safety of these induction methods remain unclear. By comparing these induction methods, we aimed to find a more efficient and safe technique for the differentiation of MSCs into nerve cells.

\section{MATERIAL AND METHODS}

\section{Animal model and treatment}

Male Sprague-Dawley rats, 4-week-old, weighing 200-300 g, were provided by Jinan Aonuo Biological Engineering Co., Ltd. Animals were acclimated to the temperature, humidity, and lighting (12 h light/dark cycle, lights on at 7:00 AM) in controlled housing rooms and were singly housed for at least one week before behavioral studies were initiated. Animals were maintained in accordance with the Guide for the Care and Use of Laboratory Animals ( $8^{\text {th }}$ edition, Institute of Laboratory Animal Resources on Life Sciences, National Research Council, National Academy of Sciences, Washington, DC, USA). All efforts were made to minimize animal suffering and to reduce the number of animals used. 


\section{Primary culture of bone marrow mesenchymal stem cells}

Rats were euthanized by $\mathrm{CO} 2$ asphyxiation, the tibiae and femora were aseptically removed and adherent soft tissue was thoroughly debrided. The marrow was drawn from the femoral marrow cavity into syringes containing low glucose Dulbecco's Modified Eagle Medium (DMEM, Gibco, Grand Islands, NY, USA) supplemented with $100 \mathrm{U}$ heparin, $100 \mathrm{U} / \mathrm{mL}$ penicillin, and $100 \mu \mathrm{g} / \mathrm{mL}$ streptomycin. Bone marrow samples were washed twice with phosphatebuffered saline (PBS) and purified by Percoll gradient. Cells were then plated in DMEM (Iow glucose) supplemented with $15 \%$ fetal bovine serum (FBS), $100 \mathrm{U} / \mathrm{mL}$ penicillin, and $100 \mu \mathrm{g} / \mathrm{mL}$ streptomycin. Cells were incubated at $37^{\circ} \mathrm{C}$ in a humidified atmosphere containing $5 \% \mathrm{CO} 2$. Nonadherent cells were removed by changing the culture medium after 2 days. After 5 passages, cells were gently harvested when $80 \%$ confluent using $0.25 \%$ trypsin in $0.1 \%$ EDTA and used for differentiation experiments.

\section{Flow cytometry}

The expression of CD90, CD44, CD105, and CD34 was detected in MSCs using flow cytometry. Cells were trypsinized at a cell density of $1.0 \times 108 / \mathrm{L}$. FITC-CD90,FITC-CD44, FITCCD105, and PE-CD34 Multicolor antibodies (BD Bioscience, San Jose, CA, USA) were added to the tube containing $0.4 \mathrm{~mL}$ bone marrow mesenchymal stem cells at $4^{\circ} \mathrm{C}$ in the dark and incubated for $1 \mathrm{~h}$. After 3 washes in PBS, the cell pellet was resuspendedin cytometry buffer and analyzed using a FACS Calibur flow cytometer (BD Bioscience).

\section{Chemically-induced differentiation}

Marrow mesenchymal stem cells at passage 5 were plated on 24-well plates and cultured in a pre-induction medium consisting of DMEM (low glucose) supplemented with 15\% FBS, $10 \mathrm{ng} /$ mL bFGF (GIBCO, PMG0031), 20 ng/mL EGF (GIBCO, PMG8043), $100 \mathrm{U} / \mathrm{mL}$ penicillin, and 100 $\mu \mathrm{g} / \mathrm{mL}$ streptomycin. After $24 \mathrm{~h}$, MSCs were cultured in induction medium, consisting of DMEM (low glucose) supplemented with $100 \mathrm{U} / \mathrm{mL}$ penicillin, $100 \mu \mathrm{g} / \mathrm{mL}$ streptomycin, and different inductors, including $1 \mathrm{mM} \mathrm{BME}$ (BME), 10-3 mM ATRA (ATRA), 0.2\% DMSO/0.2 mM BHA (DMSO/BHA), and $0.12 \mathrm{mM}$ Indomethacin/0.3 mM IBMX (Indomethacin/IBMX). Cells were culture $\mathrm{d}$ for a total period of $48 \mathrm{~h}$ at $37^{\circ} \mathrm{C}$ under a humidified atmosphere containing $5 \% \mathrm{CO} 2$.

\section{Cell viability}

MSCs were seeded at a density of 5000 cells/mL on 24-well plates (Corning Inc., Corning, NY, USA) and incubated for 10 days. The 3-(4,5-dimethylthinazol-2-yl)-2,5-diphenyl tetrazolium bromide (MTT) assay was used to evaluate cell viability and the obtained growth curves were used to establish the proliferation of cells stimulated by different inducers. Briefly, cells were counted and seeded on gelatin-coated 96-well culture plates at a density of $5 \times 10^{4}$ cells/well. After incubation with inducers or vehicle, each well was washed twice with PBS to remove the medium, $100 \mu \mathrm{L}$ MTT $(0.5 \mathrm{mg} / \mathrm{mL})$ was then added to each well, and the incubation was continued at $37^{\circ} \mathrm{C}$ for an additional $4 \mathrm{~h}$. Next, $150 \mu \mathrm{L}$ DMSO was added to each well and the absorbance $(490 \mathrm{~nm})$ was read using a microplate reader ( $\mathrm{SH}-1000$, Corona Electric, Ibaraki-ken, Japan). 


\section{Quantitative-PCR}

Total RNA was isolated by using QiagenRNeasy kit according to the manufacturer instructions. Real-time PCR primers were designed based on the NCBI database of conserved coding regions (Table 1). Each RT-PCR reaction $(10 \mu \mathrm{L})$ contained the following: SsoAdvanced SYBR GreenSuper mix $(5 \mu \mathrm{L})$, Forward primer $(300-450 \mathrm{nM})$, target reverse primer $(300-450$ $\mathrm{nM})$, cDNA synthesis reaction (100 ng), and nuclease-free PCR grade water to adjust the final volume. As a normalization control for RNA loading, parallel reactions in the same multi-well plate were performed using $\beta$-actin as a target. Quantification of gene amplification was performed following Q-PCR by determining the threshold cycle (CT) number for SYBR fluorescence within the geometric region of the semi-log plot generated during PCR. Within this region of the amplification curve, each difference of one cycle is equivalent to a doubling of the PCR product. The relative quantification of the target gene expression across treatment was evaluated using the comparative $\triangle \triangle \mathrm{CT}$ method. The CT value was determined by subtracting the most stable endogenous gene CT value from the target CT value of the sample. Calculation of $\Delta \Delta C T$ involved using target gene expression on Day 0 (Sample with the highest CT value or lowest target expression) as an arbitrary constant to be subtracted from all other CT sample values. Relative target mRNA expression was calculated as a fold change in relation to Day or Hour 0 sample and expressed as the $2^{-\Delta \Delta C t}$ value.

\begin{tabular}{lll}
\multicolumn{2}{l}{ Table 1. Primer sequences. } & \\
\hline Gene & Accession & Primer $\left(5^{\prime}-3^{\prime}\right)$ \\
\hline Nestin & NM_016701.3 & For: CCCTGAAGTCGAGGAGCTG \\
Map2 & NM_001039934.1 CTGCTGCACCTCTAAGCGA \\
Nse & NM_013509.3 & For: GCCAGCCTCGGAACAAACA \\
Gfap & NM_010277.3 & Rev: GCTCAGCGAATGAGGAAGGA \\
B-actin & For: AGGTGGATCTCTATACTGCCAAA \\
& NM_007393.3 & Rev: GTCCCCATCCCTTAGTTCCAG \\
\hline
\end{tabular}

Nestin, Mus musculus nestin (Nes); Map2, Mouse microtubule-associated protein 2 (MAP2); Nse, Mus musculus enolase 2, gamma neuronal (Eno2); Gfap, Mus musculus glial fibrillary acidic protein (Gfap); $\beta$-actin, Mus musculus actin, beta; For, forward; Rev, reverse.

\section{Western blot analysis}

Treated cells were harvested by scraping into ice-cold lysis buffer lysis buffer [20 mM Tris (pH 7.5), 150 mM NaCl, 1\% Triton X-100, 1 mM EDTA, 1 mM EGTA, 2.5 mM sodium, pyrophosphate, $1 \mathrm{mM} \beta$-glycerol phosphate, $1 \mathrm{mM} \mathrm{Na}_{3} \mathrm{VO}_{4}$, and leupeptin $\left.(1 \mu \mathrm{g} / \mathrm{mL})\right]$ and incubated on ice for 20 $\min$. These samples were centrifuged at $14,000 \mathrm{~g}$ for $10 \mathrm{~min}$ at $4^{\circ} \mathrm{C}$. The supernatant was used to determine Nestin, MAP2, and GFAP expression using antibodies from Santa Cruz Biotechnology (Santa Cruz, CA, USA). The protein concentration was determined using the BCA assay (Beyotime, Haimen, China). Equal amount of protein ( $40 \mu \mathrm{g} / \mathrm{lane})$ was subjected to SDS-PAGE and transferred to polyvinylidene fluoride membranes (Millipore Corporation, Billerica, MA, USA). Blots were blocked for $2 \mathrm{~h}$ in 5\% nonfat dry milk-TBS-0.1\% Tween 20 and then washed. Primary antibodies were incubated overnight at $4^{\circ} \mathrm{C}$ followed by a horseradish peroxidase-conjugated secondary antirabbit antibody (1:10000; Boster Co., Wuhan, China) for 2 h. Immunoreactivity was detected using 
the enhanced chemiluminescence detection reagents (Life Technologies, Carlsbad, CA, USA) by a gel imaging system (BIORAD, Hercules, CA, USA).

\section{Statistical analysis}

All data are presented as mean \pm S.D. and were analyzed by one-way analysis of variance (ANOVA), followed by Student's two-tailed t-test for comparison between two groups and Dunnett's test for three or more groups using SPSS 11.5. The significance of difference was set at $P<0.05$.

\section{RESULTS}

\section{Surface marker expression of bone marrow mesenchymal stem cells}

MSC were isolated from the bone marrow based on their capacity to adhere to the plastic flask under standard culture conditions that included DMEM medium supplemented with 15\% FBS. After 2-3 days of culture, colonies of fibroblast-like cells were visualized attached to plastic culture flasks. Isolated cells were cultured for several weeks in monolayers and used for differentiation experiments after passage 5. Flowcytometric analysis of MSC demonstrated that a higher $(P<0.05)$ percentage of these cells were positive for the MSC markers CD44, CD90, and CD105 (93.6, 85.3, and $82.6 \%$, respectively), while negative for the hematopoietic marker CD34 (5.7\%) (Figure 1).

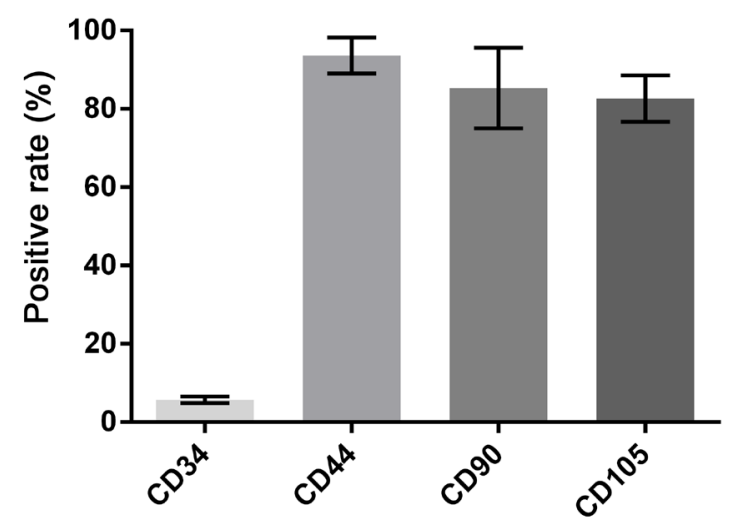

Figure 1. Surface marker expression of bone marrow mesenchymal stem cells. MSCs were harvested and stained with FITC-CD90, FITC-CD44, FITC-CD105, and PE-CD34. Values are presented as the mean \pm S.D. $(\mathrm{N}=5)$.

\section{Cellviability}

To determine the safety of these inducers, cell proliferation and viability were analyzed. Agrowth curve was first determined to ensure that cells used in the following experiments were within the exponential growth phase. As shown in Figure 2, cells proliferated rapidly on day 3-5 and cell viability remained close to $95 \%$ on the 8 th day of culture, then the number of cells began to decline. Thus, the cytotoxic effect was determined on day 3. As shown in Figure 3, the lowest viability was observed in the presence of DMSO/BHA, followed by BME, ATRA, and Indomethacin/ $\operatorname{IBMX}(75.7,85.9$, and $80.2 \%$, respectively, compared to the control group). 


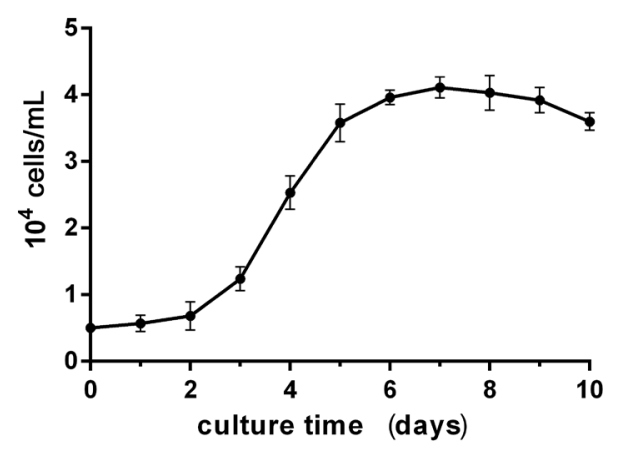

Figure 2. Growth curve of bone marrow-derived MSCs. The viability of MSCs was measured by MTT assay for 10 days. Values are expressed as mean \pm S.D. $(N=6)$.

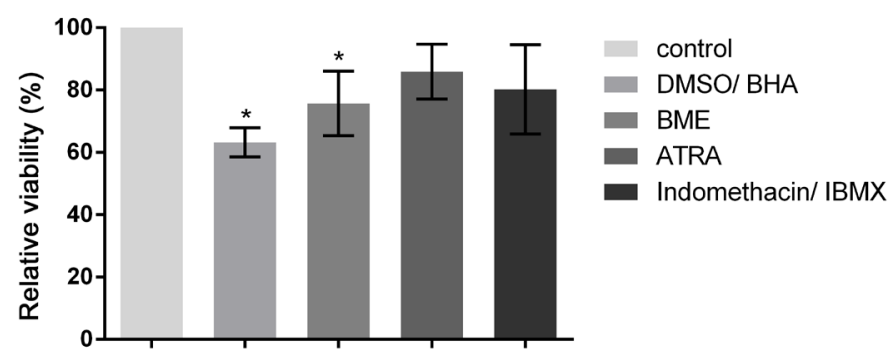

Figure 3. Viability of MSCs subjected to different inducers. MSCs were stimulated with vehicle, DMSO/BHA, BME, ATRA, or Indomethacin/IBMX, the viability was then measured by MTT assay. Values are expressed as mean \pm S.D. $(N=6)$.

\section{Expression of neural markers}

To evaluate MSC neural differentiation, the expression of neural markers (Nestin, MAP2, NSE, and GFAP) was analyzed by RT-PCR. As shown in Table 2, MSCs induced by Indomethacin/ IBMX expressed higher levels of neural markers than MSCs induced by BME, ATRA, and DMSO/ BHA. The western blot results confirmed the PCR results as shown in Figure 4. MSCs induced by $0.12 \mathrm{mM}$ Indomethacin/0.3 mM IBMX expressed higher level of MAP2 and GFAP, although nestin expression slightly decreased $(P>0.05)$. NSE expression was not affected by any of the inducers (Data not shown).

Table 2. PCR analysis of neural gene expression profile in differentiated MSCs.

\begin{tabular}{lcccc}
\hline Group & Nestin & Map2 & Nse & Gfap \\
\hline Control & 1 & 1 & 1 & 1 \\
BME & $1.1 \pm 0.2$ & $3.2 \pm 0.1$ & $2.8 \pm 0.6$ & $5.5 \pm 1.3^{*}$ \\
ATRA & $4.8 \pm 1.0$ & $2.7 \pm 0.1$ & $3.3 \pm 1.0^{*}$ & $8.3 \pm 0.6^{* *}$ \\
DMSO/BHA & $4.3 \pm 0.7$ & $2.0 \pm 0.2$ & $2.8 \pm 0.9^{*}$ & $3.6 \pm 0.6^{*}$ \\
Indomethacin/IBMX & $2.2 \pm 0.1^{*}$ & $7.3 \pm 1.4^{*}$ & $4.9 \pm 0.8^{*}$ & $11.7 \pm 1.7^{* *}$ \\
\hline
\end{tabular}

The expression of nestin, Map2, Nse, and Gfap mRNA was quantitated by real-time PCR and normalized to that of the control group. Each PCR reaction was run in triplicate. Data are expressed as the percentage of vehicle group and are presented as the mean \pm S.D. ${ }^{*} P<0.05,{ }^{* *} P<0.01$ compared with the control group. 


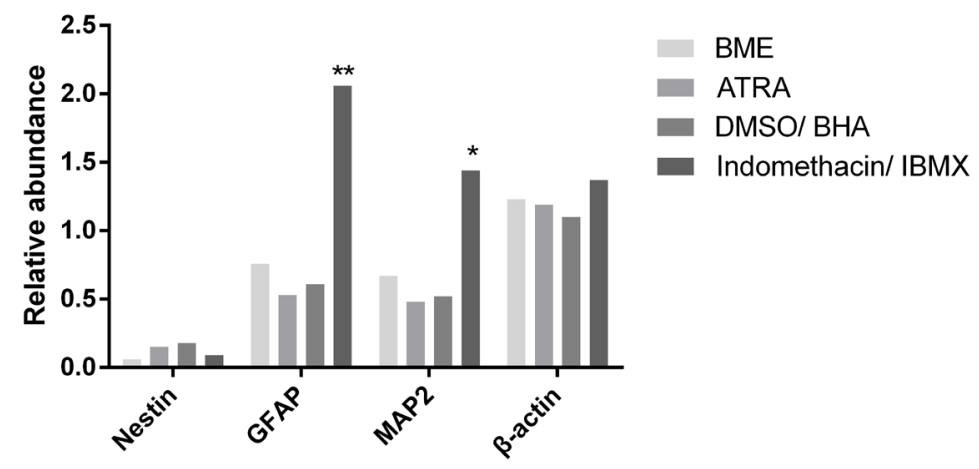

Figure 4. Western-blot analysis of neural protein expression profile in differentiated MSCs. Differentiated MSCs were stimulated by vehicle, DMSO/BHA, BME, ATRA, or Indomethacin/IBMX, the lysate were analyzed by western blotting. Data were quantified by densitometry. Data are expressed as the percentage of control and are presented as the mean \pm S.D. Each blot is representative from three to five independent experiments. ${ }^{*} P<0.05,{ }^{* *} P<0.01$ compared to the control group.

\section{DISCUSSION}

Neurogenic differentiation of bone marrow (BM) mesenchymal stem cells (MSCs) offers a new hope for patients with many neurological disorders such as Alzheimer's disease, spinal cord injury, and Parkinson's disease (Huang et al., 2012). The interest in studying this multipotent cell population largely derives from its advantages (Friedenstein et al., 1987). For instance, MSCs can be easily harvested for autologous transplantation and do not involve ethical issues (Grayson et al., 2015). Although both undifferentiated and differentiated MSCs have been used for cell transplantation in neurological diseases, in vitro differentiation prior to transplantation may be safer than in vivo implantation (Yaghoobi et al., 2008). Thus, determining the ideal conditions to obtain neural cells is necessary.

In the present study, MSCs were isolated from BM based on their capacity to adhere to the plastic substrate under monolayer culture conditions, then identified by flow cytometry. BM-derived MSCs express surface markers, including CD90, CD44, CD105, and CD166, and lack hematopoietic lineage markers, CD34 and CD45 (Kala et al., 2012; Xue et al., 2015). A high proportion of cells expressed MSC-specific cell type markers, including CD34, CD44, CD90, and CD105, but did not express CD34, indicating that the cells in this study were of mesenchymal origin and pure.

Reports indicate that the neural differentiation of MSCs is achieved by using different experimental protocols, Protocols include using chemical agents like $\beta$-mercaptoethanol, tretinoin, a mix of DMSO and butylatedhydroxyanisole, or a mix of IBMX and indomethacin (Fu et al., 2008; Qian et al., 2010; Tse et al., 2010; Kaka et al., 2012). We then compared the effectiveness and the safety of these induction methods. MSCs induced by Indomethacin/IBMX expressed the highest mRNA levels of nestin, Map2, Nse, and Gfap. Similarly, MAP2 and GFAP protein levels expressed by MSCs induced by Indomethacin/IBMX were higher than those of MSCs stimulated by other chemicals. These results indicated the effectiveness of Indomethacin/IBMX to induce MSCs toward the neural linage. The cytotoxicity of these inducers was also evaluated by MTT assay. The results showed that Indomethacin/IBMX and ATRA did not significantly affect the proliferation and viability of MSCs, while DMSO/BHA and BMA affected cell viability. Taken together, neurogenic 
differentiation of MSCs induced by Indomethacin/IBMX is more effective and safer than induction using ATRA, DMSO/BHA, and BME.

\section{Conflicts of interest}

The authors declare no conflict of interest.

\section{REFERENCES}

Chen L, Qiu R and Xu Q (2014). Mesenchymal stem cell therapy for neurodegenerative diseases. J. Nanosci. Nanotechnol. 14: 969-975.

Friedenstein AJ, Chailakhyan RK and Gerasimov UV (1987). Bone marrow osteogenic stem cells: in vitro cultivation and transplantation in diffusion chambers. Cell Tissue Kinet. 20: 263-272.

Fu L, Zhu L, Huang Y, LeeT D, et al. (2008). Derivation of neural stem cells from mesenchymalstem cells: evidence for a bipotential stem cell population. Stem Cells Dev. 17: 1109-1121.

Fukuda K (2001). Development of regenerative cardiomyocytes from mesenchymal stem cells for cardiovascular tissue engineering. Artif. Organs 25: 187-193.

Grayson WL, Bunnell BA, Martin E, Frazier T, et al. (2015). Stromal cells and stem cells in clinical bone regeneration. Nat. Rev. Endocrinol. 11: 140-150.

Huang B, Tabata Y and Gao JQ (2012). Mesenchymal stem cells as therapeutic agents and potential targeted gene delivery vehicle for brain diseases. J. Control Rel. 162: 464-473.

Kaka GR, Tiraihi T, Delshad A, Arabkheradmand J, et al. (2012). In vitro differentiation of bone marrow stromal cells into oligodendrocyte-like cells using triiodothyronine as inducer. Int. J. Neurosci. 122: 237-247.

Kala S, Kaushik R, Singh KP, Kadam PH, et al. (2012). In vitro culture and morphological characterization of prepubertal buffalo (Bubalus bubalis) putative spermatogonial stem cell. J. Assist. Reprod. Genet. 29: 1335-1342.

Kristjansson B and Honsawek S (2014). Current perspectives in mesenchymal stem cell therapies for osteoarthritis. Stem Cells Int. 2014: 194318.

Mizukami H and Yagihashi S (2014). Exploring a new therapy for diabetic polyneuropathy - the application of stem cell transplantation. Front. Endocrinol. 5: 45.

Notara M and Ahmed A (2012). Benign prostate hyperplasia and stem cells: a new therapeutic opportunity. Cell Biol. Toxicol. 28: 435-442.

Qian DX, Zhang HT, Ma X, Jiang XD, et al. (2010). Comparison of the efficiencies of three neural induction protocols in human adipose stromal cells. Neurochem. Res. 35: 572-579.

Swart JF and Wulffraat NM (2014). Mesenchymal stromal cells for treatment of arthritis. Best Pract. Res. Clin. Rheumatol. 28: 589-603.

Tatullo M, Marrelli M and Paduano F (2015). The regenerative medicine in oral and maxillofacial surgery: the most important innovations in the clinical application of mesenchymal stem cells. Int. J. Med. Sci. 12: 72-77.

Tse KH, Sun M, Mantovani C, Terenghi G, et al. (2010). In vitro evaluation of polyester-based scaffolds seeded with adipose derived stem cells for peripheral nerve regeneration. J. Biomed. Mater. Res. A 95: 701-708.

Widgerow AD, Salibian AA, Lalezari S and Evans GR (2013). Neuromodulatory nerve regeneration: adipose tissue-derived stem cells and neurotrophic mediation in peripheral nerve regeneration. J. Neurosci. Res. 91: 1517-1524.

Woodbury D, Schwarz EJ, Prockop DJ and Black IB (2000). Adult rat and human bone marrow stromal cells differentiate into neurons. J. Neurosci. Res. 61: 364-370.

Wu KH, Wu HP, Chan CK, Hwang SM, et al. (2013). The role of mesenchymal stem cells in hematopoietic stem cell transplantation: from bench to bedsides. Cell Transplant. 22: 723-729.

Xue K, Xia W, Zhang X, Qi L, et al. (2015). Isolation and identification of stem cells in different subtype of cartilage tissue. Expert Opin. Biol. Ther. 15: 623-632.

Yaghoobi MM and Mahani MT (2008). NGF and BDNF expression drop off in neurally differentiated bone marrow stromal stem cells. Brain Res. 1203: 26-31.

Zhang Y, Xu L, Wang S, Cai C, et al. (2014). Concise review: differentiation of human adult stem cells into hepatocyte-like cells in vitro. Int. J. Stem Cells 7: 49-54. 